\title{
Assessing the Malignancy of Hepataocellular Carcinoma in Cirrhosis Using the Hepatocellular Contrast Agent Gd-EOB-DTPA and Correlation with the Cytometric DNA Index
}

\author{
R. Röttgen ${ }^{1}$, S. Kobi ${ }^{1}$, C. Grieser ${ }^{1}$, F. Streitparth ${ }^{1}$, H. Al-Abadi ${ }^{2}$, A. Duerr ${ }^{2}$, W. Faber ${ }^{2}$, J. Pratschke ${ }^{3}$, \\ P. Neuhaus ${ }^{2}$, B. Hamm ${ }^{2}$, L. Lüdemann ${ }^{4}$ \\ ${ }^{1}$ Klinik für Radiologie, Charite’ Campus Virchow Augustenburger Platz 1, Berlin, Germany \\ ${ }^{2}$ Klinik für Allgemein-Visceral- und Transplantationschirurgie, Charite’ Campus Virchow Augustenburger Platz 1, Berlin, Germany \\ ${ }^{3}$ Klinik für Visceral-, Transplantations- und Thoraxchirurgie, Universitätsklinikum Innsbruck, Innsbruck, Austria \\ ${ }^{4}$ Abteilung für Strahlenphysik, Universitätsklinikum Essen, Essen, Germany \\ Email: rainer.röttgen@charite.de, sikocope@gmx.de, christian.grieser@charite.de, florian streitparth@charite.de, \\ hussein.al-abadi@charite.de, angelika.duerr@charite.de,wladimir.faber@charite.de,johann.pratschke@i-med.ac.at, \\ peter.neuhaus@charite.de, bernd.hamm@charite.de, lutz.luedemann@uk-essen.de
}

Received November 26, 2012; revised December 27, 2012; accepted January 5, 2013

\begin{abstract}
Aim: Investigation whether the signal intensity of hepatocellular carcinoma (HCC) on dynamic magnetic resonance imaging (MRI) with hepatocellular contrast medium (CM) correlates with the histologic malignancy grade and cytometric DNA index. Material and Methods: MRI at 1.5 T with hepatocellular CM Gd-DTPA-EOB of 63 patients (52 men, 11 women; $64.0 \pm 8.5$ years) with HCC in cirrhosis. SI of the tumor and liver tissue in sequences (T1, T1fs, T2fs, T1fs + CM (arterial, venous, late venous phase) were correlated like the asymmetry coefficient of the SI in the arterial and venous phases respective the native and late venous phases of the tumor with the histologic malignancy grade and DNA index indicating the grade of abnormal DNA steam line respective aneuploid DNA. Results: SI did not significantly correlate with the histologic malignancy grade or DNA index for precontrast or postcontrast images. There is a significant correlation for the asymmetry coefficient of the SI in the arterial and venous phases with the DNA-index (p values of 0.00089 (Pearson) and 0.0082 (Spearman). Conclusion: A highly arterialized hepatocellular carcinoma with rapid washout in the venous phase corresponds to a higher malignant potential. These findings suggest that the MR parameters investigated here may predict the malignant potential and prognosis of HCC before surgery.
\end{abstract}

Keywords: Hepatocellular Carcinoma; DNA Index; DNA Cytometry; Malignancy Grading

\section{Introduction}

Magnetic resonance imaging (MRI) with contrast medium is currently the best imaging modality for the detection and characterization of hepatocellular carcinoma (HCC) [1,2]. It is based on the signal enhancement pattern after administration of contrast medium, particularly extracellular or tissue-specific hepatobiliary agents [2]. Gd-EOB-DTPA (Primovist ${ }^{\circledR}$, Bayer Healthcare, Berlin, Germany) is an MR contrast agent combining the properties of extracellular and hepatocellular agents. This means that images can be acquired both in the dynamic phase when the contrast agent is still in the intravascular space and in the hepatobiliary phase when the agent is metabolized by the hepatocytes. Accumulation in heaptocytes occurs within a few minutes of injection, enabling clear delineation of a HCC from healthy liver tis- sue.

Dynamic MRI with a fast T1-weighted gradient echo sequence obtained during the arterial (Figure 1), portal venous (Figure 2), and late venous phases (Figure 3) after intravenous bolus administration of the contrast medium has been shown to be useful for characterizing HCC, which typically exhibits early arterial enhancement because it is highly arterialized [3]. Accumulation of contrast medium indicates a tumor of hepatocellular origin. In the routine clinical setting, however, differentiation of HCC from regenerative nodules in the cirrhotic liver continues to pose a challenge. In general, though, the use of morphologic imaging criteria allows adequate characterization of an intrahepatic tumor, and histologic confirmation of the diagnosis of HCC is generally not necessary before surgery. 


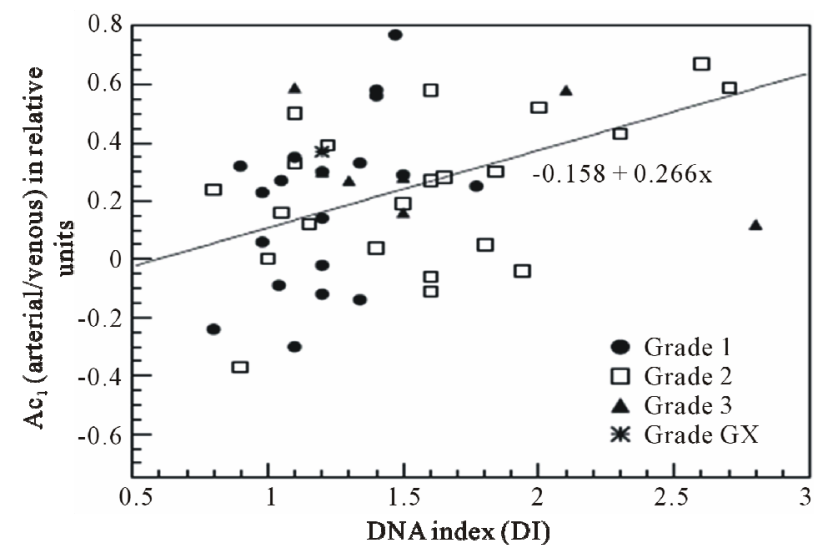

Figure 1. Asymmetry coefficient $A C_{\text {art-ven }}$ is plotted against the DNA index. Different symbols indicate the different tumor grades: filled black circle-grade 1; open squaregrade 2; filled black square-grade 3 ; asterisk-grade $X$. The solid line represents the linear fit of the correlation between the two parameters. The result of the linear fit is described by the following equation: $\mathrm{AC}_{\text {art-ven }}=-\mathbf{0 . 1 5 8}+$ $0.266 *$ DNA index.

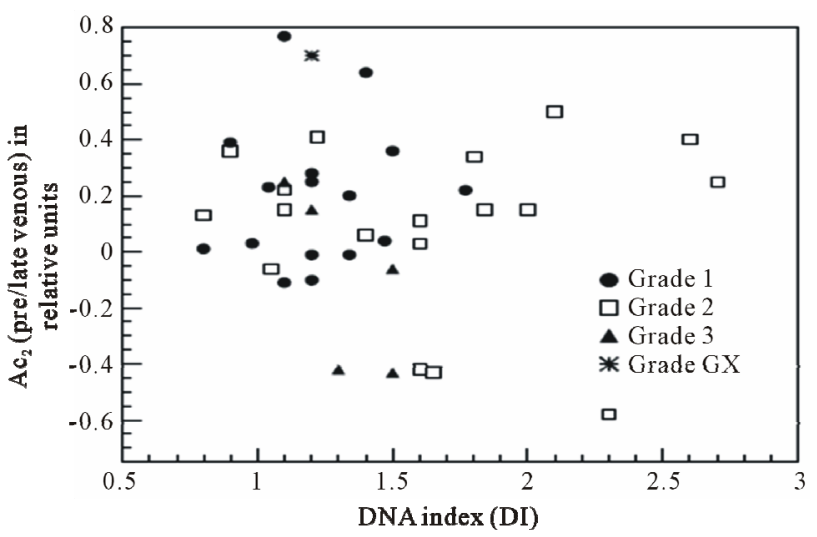

Figure 2. Asymmetry coefficient $\mathrm{AC}_{\text {pre-late venous is plotted }}$ against the DNA index. Different symbols indicate the different tumor grades: filled black circle-grade 1; open square — grade 2; filled black square-grade 3; asteriskgrade $X$. There is no correlation between the two parameters.

Accurate diagnostic characterization and differentiation of a malignant liver tumor is done using histologic grading or DNA cytometry. The latter may have important implications for treatment as it enables objective and reliable grading of the malignant potential. Photometric DNA measurement is based on the quantitative determination of the DNA content of cell nuclei [4]. The material for DNA analysis can be obtained by fine needle aspiration and may provide important additional information on the biologic behavior of a tumor that may alter the therapeutic strategy and determines the prognosis. Tumor cells are characterized by numerical and/or structural chromosome aberrations. Severe chromosomal aberration identified as significant by DNA cytometry is

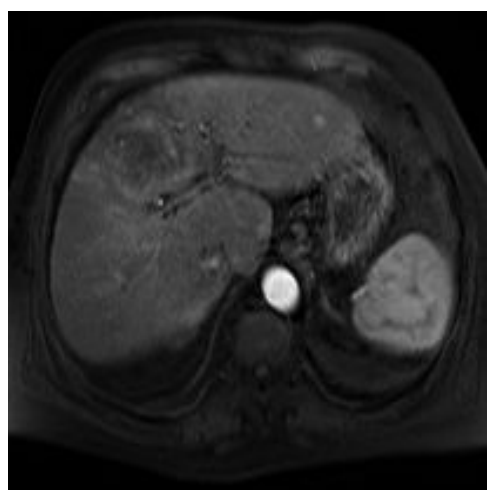

Figure 3. HCC in liver segment 4 during the arterial phase; fat-saturated T1-weighted image acquired approx. $20 \mathrm{~s}$ after the start of intravenous contrast medium administration. There is increased peripheral enhancement suggesting high arterial perfusion of the lesion.

referred to as DNA aneuploidy and is an indicator of the malignant potential of a tumor. Hence, DNA ploidy or the DNA index has been identified to be an important prognostic indicator in patients who underwent liver transplantation because of HCC in cirrhosis [4-13].

Jonas et al. showed DNA ploidy to be a useful prognostic factor in patients who received a liver transplant for HCC in cirrhosis, demonstrating that its predictive value is superior to tumor staging. In this study, aneuploidy, or a high DNA index, indicated a poorer prognosis in liver transplant recipients than diploid and polyploid carcinomas with a low DNA index [14].

The purpose of the study is to evaluate, if an assessing of the malignancy of hepataocellular aarcinomas in cirrhosis using MRI with hepatocellular contrast agent will be possible.

\section{Patients and Methods}

Sixty-three patients with histologically proofed HCC in cirrhosis who underwent MRI of the liver with the heaptocellular MR contrast agent Gd-EOB-DTPA were retrospectively included in the study. All of them underwent resection or liver transplant. The patients had a mean age of $63.0 \pm 10.0$ years (52 men, $64.0 \pm 8.5$ years, range 43 84; 11 women, $58.3 \pm 14.9$ years, range 32 - 82). Pathohistologic grading of the patients included in the study yielded 26 well differentiated (G1), 28 moderately differentiated (G2), and 8 poorly differentiated HCCs (G3). In one HCC, the degree of differentiation could not be determined (GX).

\section{DNA Cytometry}

DNA cytometry was available for 52 HCCs.It is measured by up to 300 tumor cell nuclei, sometimes more, and 30 located in the same preparation as lymphocytes and leukocytes were used as analytical reference cells. 
The DNA content determined by means of these nuclei singlecellabsorptionphotometry. The measurement was carried out in the fine-scanning method using a microscope cytophotometer (Ahrens Medical Supply Tecnic, argteheide, Germany). The control of the scanning table, the measuring process and the registration of the absorption programmatically were effected by process computer. The measured data of the respective total extinktion were printed along with the mean, standard deviation, variance and coefficient of variation through a telephoto type. The measurement of the integrated optical density of nuclei is coupled interactive on the monitor of a conventional microscope with a PC-based TV image analysis system. The microscope is equipped with a color TV camera and appropriate interference filters. Within the relevant cell population of at least 300 cell nuclei are measured randomly assigned. The measurement is done automatically by clicking the relevant nuclei with a mouse on the screen. A DNA histogram. determined per measurement DNA content of about 300 cell nuclei is combined in a cytophotogramm and as an absolute value in AU (arbitraryunits) specified. The number of cytomorphometric nuclei measured is denoted by " $n$ ". The determination of the $2 \mathrm{c}$ value, i.e., of the DNA-content corresponding to a normal diploid set of chromosomes occurs in lymphocytes or leukocytes as a calibration diploid cell population. The so-called standard cells (standard calibration cell population) have to be set in the same manner as the measured nuclei of the hepatocytes Hepatozytenkerne, hydrolyzed and stained according to Feulgen. The doubling of the 2 c-values is given in the cytophotogram with $4 \mathrm{c}$ and corresponds to a tetraploid, a further doubling (quadrupling) a oktaploiden (8 c) set of chromosomes. The diagnosis of the DNA histograms are qualitatively according to the ESCAP Consensus Report in the categories of DNA diploid, DNA polyploid and DNA-aneuploid and is performed according to the following criteria: diploid DNA: DNA base line (STL) > $1.80 \mathrm{c}<2.20$ c polyploid DNA: DNA base line (STL) $>$ $1.80 \mathrm{c}<2.20$ and $\mathrm{c}>3.60 \mathrm{c}<4.40$ c.DNA aneuploid: abnormal DNA stem line (STL) $<1.80 \mathrm{c}>2.20$, or c $<$ $3.60 \mathrm{c}>4.40 \mathrm{c}$ and/or values $>9 \mathrm{c}$.

\section{MRI-Examination}

MRI examinations were performed on a 1.5-Tesla Genesis Signa MR scanner (General Electric Medical Systems, Wisconsin, USA) using an 8-channel surface receive only coil. The following pulse sequences were analyzed:

- Native breath-hold T1-weighted 2D gradient echo (GRE) sequence (fast spoiled gradient echo, FSGE); repetition time (TR): $212 \mathrm{~ms}$, echo time (TE): $4.6 \mathrm{~ms}$, flip angle (FA): $80^{\circ}$, slice thickness (SL): $8 \mathrm{~mm}$, interslice gap: $0 \mathrm{~mm}$, field of view (FOV): $36 \times 36 \mathrm{~cm}$, image matrix: $512 \times 512$, acquisition rate: 24 slices in
$15 \mathrm{~s}$.

- Breath-hold axial T1-weighted 3D GRE sequence with fat saturation (FS); TR: $119 \mathrm{~ms}$, TE: $6.9 \mathrm{~ms}$, FA: $70^{\circ}$, SL: $8 \mathrm{~mm}$, FOV: $36 \times 36 \mathrm{~cm}$, image matrix: 512 $\mathrm{x}$ 512, acquisition rate: 24 slices in $40 \mathrm{~s}$.

- 2D fast T2-weighted spin echo sequence (fast/turbo spin echo, FSE) with FS; TR: $2100 \mathrm{~ms}$, TE: $90 \mathrm{~ms}$, FA: $90^{\circ}$, turbo factor, ETL: 21, axial, SL: $8 \mathrm{~mm}$, gap: $0 \mathrm{~mm}$, FOV: $36 \mathrm{~cm} \times 36 \mathrm{~cm}$, image matrix: $512 \times$ 512, acquisition rate: 28 slices in 1 min $9 \mathrm{~s}$.

- Dynamic breath-hold axial 3D T1-weighted sequence; arterial and venous phase (2 phases) after manual IV bolus administration of $0.025 \mathrm{mmol} / \mathrm{kg}$ of Gd-EOBDTPA $(0.1 \mathrm{ml})$. TR: $10.3 \mathrm{~ms}, \mathrm{TE}: 5.2 \mathrm{~ms}, \mathrm{FA}=15^{\circ}$, ETL: 59, SL: $5 \mathrm{~mm}$, gap: $0 \mathrm{~mm}$, FOV: $36 \times 36 \mathrm{~cm}$, image matrix: $256 \times 256$, acquisition rate: 2 3D datasets with 40 slices in $20 \mathrm{~s}$ per phase. The arterial phase was acquired $20 \mathrm{~s}$ after contrast medium administration, the venous phase $50 \mathrm{~s}$ after administration.

- 2D breath-hold T1-weighted GRE sequence (FSGE) approx. $20 \mathrm{~min}$ after manual IV bolus administration of $0.025 \mathrm{mmol} / \mathrm{kg}$ of Gd-EOB-DTPA $(0.1 \mathrm{ml})$. TR: $212 \mathrm{~ms}$, TE: $4.6 \mathrm{~ms}, \mathrm{FA}: 80^{\circ}$, SL: 8mm, gap: $0 \mathrm{~mm}$, FOV: $36 \times 36 \mathrm{~cm}$, image matrix: $512 \times 512$, FOV: $256 \times 205$, acquisition rate: 24 slices in $15 \mathrm{~s}$.

The MRI datasets were analyzed on a workstation (General Electrics picture archiving and communication system-GE-PACS, GE Healthcare, USA, Software Centricity RA 1000, version 2027435-091 using two EIZO RadiForce GS 220 monitors, gray-scales, EIZO Nanao, Hakui Corporation, Japan). Signal intensities (SI) with standard deviations (SD) were measured in regions of interest (ROIs) placed in the HCC and in representable noncancerous liver tissue on the standardized pulse sequences described above. SI in the tumor ROI was measured over the entire tumor area visualized in the slice with the largest axial tumor extent and in an area of approx. $40 \mathrm{~mm}^{2}$ in the liver tissue.

For standardization of the measured SIs, ratios of SI in the HCC and noncancerous liver tissue were calculated, referred to as standardized signal intensity (I) below.

$$
I=\frac{S I \text { (tumor) }}{S I \text { (liver) }}
$$

Dynamic contrast enhancement was calculated using the asymmetry coefficient $A C_{\text {art-ven, which describes the }}$ difference in standardized SIs between the arterial and venous phase.

$$
A C_{\text {art-ven }}=\frac{S I(\text { art })-S I(\text { ven })}{0.5(S I(\text { art })+S I(\text { ven }))}
$$

The asymmetry coefficient $A C_{\text {late-ven }}$ describes the difference in standardized SIs before contrast medium ad- 
ministration and the late venous phase (20 min after IV contrast medium administration).

$$
A C_{\text {pre-late-ven }}=\frac{I(\text { pre })-I(\text { post })}{0.5(I(\text { pre })+I(\text { post }))}
$$

The fact that only these two asymmetry coefficients could be calculated is due to the pulse sequences that were available for analysis: Postcontrast images in the arterial and venous phase were acquired with the T1weighted 3D sequence, while nonenhanced images and the late venous phase were acquired using the T1weighted 2D sequence. This is why no asymmetry coefficients could be calculated for nonenhanced SI and the arterial phase or for the arterial or venous phase and the late venous phase.

The standardized signal intensities, I, of HCCs determined from the above-described sequences and the two asymmetry coefficients $\left(A C_{\text {art-ven }}, A C_{\text {late-ven }}\right)$ were correlated with histopathologic grades in all 63 patients and with the cytometric DNA index in 52 patients.

\section{Results}

Analysis by degree of HCC differentiation did not reveal any significant correlations for the standardized signal intensities on the unenhanced pulse sequences and the cytometric DNA index for any of the groups. The same was true for the standardized signal intensities measured on the contrast-enhanced images and their correlation with the DNA indices for any of the pathohistologic HCC grades.

With increasing DNA indices, we saw a tendency for higher standardized signal intensities in the arterial phase and for lower standardized signal intensities in the venous phase.

In contrast, significant correlations were found for $\mathrm{AC}_{\text {art-ven }}$ and the DNA index using both Pearson's correlation analysis (linear) $(\mathrm{p}=0.00089)$ and the Spearman rho (nonlinear) $(\mathrm{p}=0.0082)$. The second asymmetry index investigated, $A C_{\text {pre-late-ven, did not significantly corre- }}$ late with the DNA index $(p=0.67 ; p=0.40)$. No significant correlation of the asymmetry index with the tumor grade was found.

The Figures $\mathbf{4}$ and $\mathbf{5}$ illustrate the relationships between the two asymmetry coefficients, $A C_{\text {art-ven }}$ and $A C_{\text {pre-late-venous, }}$ and the DNA index.

\section{Discussion}

We found no correlation between the MR signal intensities of HCCs on the pulse sequences investigated and the tumor stage or cytometric DNA index for nonenhanced images or the arterial, venous, or late venous phases of the dynamic series; however, we saw a tendency for increasing DNA indices to be associated with

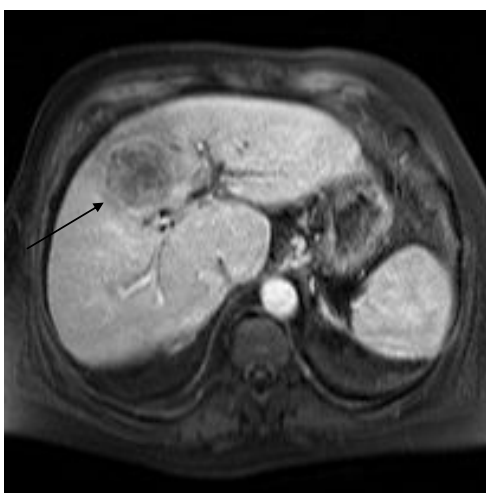

Figure 4. HCC in liver segment 4 during the venous phase; fat-saturated T1-weighted image acquired approx. $50 \mathrm{~s}$ after the start of intravenous contrast medium administration. The signal intensity of the lesion is lower than that of surrounding liver tissue, which indicates rapid washout/reflux in the venous phase.

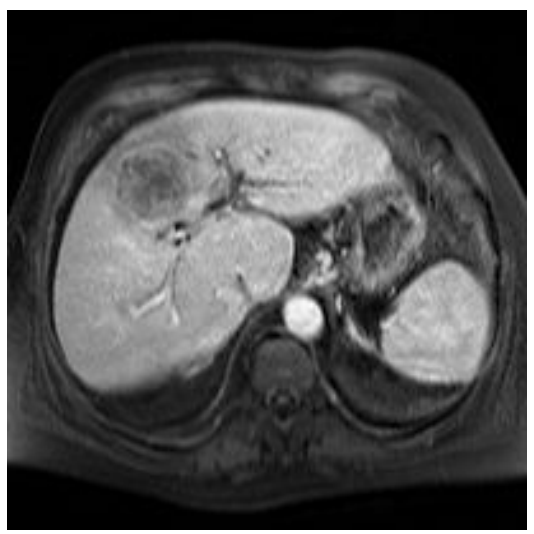

Figure 5. HCC in liver segment 4 during the venous phase; fat-saturated T1-weighted image acquired approx. $50 \mathrm{~s}$ after the start of intravenous contrast medium administration. The signal intensity of the lesion is lower than that of surrounding liver tissue, which indicates rapid washout/reflux in the venous phase.

higher standardized signal intensities in the arterial phase and lower intensities in the venous phase. In contrast, we found a significant correlation between asymmetry coefficient $A C_{\text {art-ven }}$ and the DNA index but not for the second asymmetry coefficient we investigated, i.e., the one between signal intensities on unenhanced images and those measured in the late venous phase. These results suggest that the malignant potential of HCC, expressed in the DNA index, increases with an increasing difference in standardized signal intensities between the arterial and venous phases, as indicated by increasing values of asymmetry coefficient $A C_{\text {art-ven }}$.

In terms of function, this means that strong arterializetion of a tumor with rapid washout in the venous phase corresponds to a higher DNA index and hence a higher malignant potential. Conversely, a lower value for $A C_{\text {art-ven }}$ corresponds to a lower DNA index or malignant poten- 
tial.

We attribute this result to the assumption that a high malignant potential, as indicated by a high DNA index, is associated with enhanced angiogenesis and results in tumors with a larger number of arteries and veins. This assumption would explain the stronger arterial and weaker venous enhancement we saw in tumors with a high DNA index, as opposed to those with a lower DNA index. The presence of more vessels in a tumor means that contrast medium inflow and outflow is both faster and more pronounced.

Conversely, our initial hypothesis that contrast-enhanced MRI using Gd-EOB-DTPA would also improve the characterization of HCC in cirrhosis with regard to histopathological tumor grades was not confirmed here. We found no statistically significant associations between standardized signal intensities in the arterial, venous and late phases of the dynamic contrast-enhanced series and the histopathologic tumor grade. This disappointing outcome might also in part be due to the fact that HCC is a heterogeneous group with mixed types and overlap between the different histological grades, making it difficult at times to assign a tumor to a specific histologic grade.

Based on the results presented here, the dynamic contrast behavior of HCC might supplement preoperative MRI, providing useful diagnostic information that goes beyond the Milan criteria in evaluating candidates for liver transplant. Enhancement patterns might provide clues about the malignant potential and the prognosis that cannot be derived from merely evaluating the morphologic imaging appearance of HCC. It might also be speculated that our results on the potential role of con-trast enhancement also apply to other, nonspecific contrast agents.

Imaging detection of HCC in cirrhosis has been investtigated in many studies [1,15-20]. Schneider et al. and Hentrich et al. demonstrated the significance of contrast-enhanced dynamic MRI using the hepatobiliary contrast agent Gd-BOPTA (Multi-Hance ${ }^{\circledR}$ ) for the detection and differentiation of benign and malignant hepatic lesions [21]. Hentrich et al. showed that late enhancement is related to cellular differentiation with moderately differentiated HCC exhibiting significantly stronger uptake of contrast medium than poorly differentiated HCC. Horigome et al. compared MRI including dynamic contrast-enhanced imaging using Gd-DTPA (Magnevist ${ }^{\circledR}$ ) with the histopathologic grades of tumor differenttiation and showed that detection by MRI improves with poorer differentiation and larger tumor size [22]. Jonas et $a l$. investigating patients who received a liver transplant for HCC identified DNA ploidy as a valuable prognostic factor that is superior to tumor stage and other prognostic factors. In this study of patients who received a liver transplant because of HCC, aneuploidy indicated a poorer prognosis compared with diploid or polyploid HCCs [23]. In a univariate analysis, the same study group also identified the cytometric DNA index as a prognostic parameter in liver transplant recipients after HCC in cirrhosis in addition to the Milan criteria, the histopathologic tumor grade, vascular invasion, and the cytometric DNA index. A multivariate analysis identified the DNA index and vascular invasion as prognostic parameters [10].

The Milan criteria, which are used as a basis for selecting patients with HCC in cirrhosis for liver transplantation, take into account the number and size of lesions. In addition, information on differentiation and malignancy of an HCC would be also useful. Liver transplant recipients meeting the Milan criteria have a mean 5 -year survival rate of $62 \%$ to $70 \%$ when the degree of differentiation of HCC is not taken into account. Patients not meeting these criteria have poorer survival rates. Several histopathologic studies of explanted livers revealed microvascular tumor invasion as an independent negative predictor of survival after liver transplant. These studies also showed a significantly higher incidence of microvascular invasion with increasing tumor size $[11,24,25]$. The risk of microvascular invasion is greater than $50 \%$ for patients with HCC $>5 \mathrm{~cm}$.

Other factors, apart from the Milan criteria, that have been found to influence survival are the histopathologic tumor grade, vascular invasion, and DNA cytometry (DNA ploidy, DNA index) $[10,11,23,25]$. The signifycant association we found between the arterial-venous asymmetry coefficient, i.e., the ratio of contrast enhancement in the arterial phase to that in the venous phase, and the DNA index suggests that a dynamic MRI study of the liver using the hepatocellular contrast agent Gd-EOBDTPA provides information on malignancy or the biological aggressiveness of HCC. Used in patients with HCC in cirrhosis, this asymmetry coefficient might provide supplementary information for selecting the surgical procedure (extent of resection/liver transplant) or serve as a prognostic factor.

Additional studies-ideally in larger patient populations-are needed to confirm our findings. The Milan criteria might then be supplemented by the preoperatively determined MRI criterion of the asymmetry coefficient describing the ratio of arterial enhancement to venous washout following administration of a hepatocytic contrast agent.

\section{Summary}

Our study investigating signal intensities and asymmetry coefficients determined from preoperative MR images obtained using the hepatocellular contrast agent GdEOB-DTPA in patients with hepatocellular carcinoma and cirrhosis revealed a statistically significant relation- 
ship between the asymmetry coefficient of signal enhancement in the arterial and venous phases and the cancer's malignant potential as reflected in the DNA index. The asymmetry coefficient thus has the potential to enable preoperative assessment of the malignant potential of HCC and its prognosis. Preoperative assessment of the malignancy or aggressiveness of HCC using this MRI asymmetry coefficient might be a useful supplement to other imaging criteria such as lesion size, number, and site.

\section{REFERENCES}

[1] M. Scharitzer, "Characterization of Hepatocellular Tumors: Value of Mangafodipir-Enhanced Magnetic Resonance Imaging," Journal of Computer Assisted Tomography, Vol. 29, No. 2, 2005, pp. 181-190. doi:10.1097/01.rct.0000156397.51640.a1

[2] T. J. Vogl, K. Eichler, S. Zangos, M. Mack and R. Hammerstingl, "Das Hepatozelluläre Karzinom: Rolle der Bildgebung zur Detektion, Therapieplanung und Therapiekontrolle," Fortschr Röntgenstr, Vol. 174, No. 11, 2002, pp. 1358-1368. doi:10.1055/s-2002-35349

[3] Y. Tang and Y. Yamashita, "Detection of Hepatocellular Carcinoma Arising in Cirrhotic Livers: Comparison of Gadolinium- and Fermoxides-Enhanced MR Imaging," American Journal of Roentgenology, Vol. 172, No. 6, 1999, pp. 1547-54

[4] F. W. Kolkmann, "Die Bedeutung der Statischen DNAZytometrie in Diagnostik und Therapie: 21. Interdisziplinäres Forum der Bundesärztekammer, Fortschritt und Fortbildung in der Medizin,” Deutsches Ärzteblatt, Vol. 94, No. 14, 1997, p. 924.

[5] H. Abou-Rebyeh, V. Borgmann, R. Nagel and H. AlAbadi, "DNA Ploidy Is a Valuable Predictor for Prognosis of Patients with Resected Renal Cell Carcinoma," Cancer, Vol. 92, No. 9, 2001, pp. 2280-2285. doi:10.1002/1097-0142(20011101)92:9<2280::AID-CNC R1574>3.0.CO;2-2

[6] H. Abou-Rebyeh, H. Al-Abadi, S. Jonas, I. Rotter, W. O. Bechstein and P. Neuhaus, "DNA Analysis of Cholangiocarcinoma Cells: Prognostic and Clinical Importance," Cancer Detection and Prevention, Vol. 26, No. 4, 2002, pp. 313-319. doi:10.1016/S0361-090X(02)00057-0

[7] H. Al-Abadi, S. Jonas and P. Neuhaus, "DNA-Content and Survival Rate of Patients with Hepatocellular Carcinoma," Cancer Detection and Prevention, Vol. 22, No. 1, 1998, p. 180

[8] H. Al-Abadi and R. Nagel, "Zellkern-DNA-Analyse bei Nierenzellkarzinomen unter Berücksichtigung des Morphologischen Malignitätsgrade,” Aktuel Urol, Vol. 18, No. 3, 1987, pp. 137-141. doi:10.1055/s-2008-1061435

[9] T. Blöndal and J. Ponten, "DNA Ploidy in Small Cell Carcinoma of the Lung," Anticancer Research, Vol. 3, No. 1, 1983, pp. 47-52

[10] S. Jonas, H. Al-Abadi, Ch. Benckert, A. Thelen, M. Hippler-Benscheid, K. Saribeyoglu, B. Radtke, J. Prats- chke and P. Neuhaus, "Prognostic Significance of the DNA-Index in Liver Transplantation for Hepatocellular Carcinoma in Cirrhosis,” Annals of Surgery, Vol. 250, No. 6, 2009, pp. 1008-1013. doi:10.1097/SLA.0b013e3181b2b195

[11] S. Jonas, W. O. Bechstein, T. Steinmüller, R. Herrmann, C. Radke, T. Berg, U. Settmacher and P. Neuhaus, "Vascular Invasion and Histopathologic Grading Determine Outcome after Liver Transplantation for Hepatocellular Carcinoma in Cirrhosis," Hepatology, Vol. 33, No. 5, 2001, pp. 1080-1086. doi:10.1053/jhep.2001.23561

[12] H. Gustafson, B. Tribukait and P. L. Eposti, "DNA Profile and Progression in Patient with Superficial Bladder Tumors,” Urological Research, Vol. 10, No. 1, 1982, pp. 13-18. doi:10.1007/BF00256518

[13] B. Ljundberg, G. Cewrien, R. Stenling and A. Zetterberg, "The Prognostic Significance of Nuclear DNA Content in Renal Cell Carcinoma,” Journal of Urology, Vol. 135, No. 2, 1986, pp. 422-426

[14] S. K. Kim, H. K. Lim and W. J. Lee, "Detection of Hepatocellular Carcinoma: Comparison of Dynamic Three-Phase Computed Tomography Images and Four-Phase Computed Tomography Images Using Multidetector Row Helical Computed Tomography," Journal of Computer Assisted Tomography, Vol. 26, No. 5, 2002, pp. 691-698. doi:10.1097/00004728-200209000-00005

[15] C. R. Habermann, F. Weiss, M. Hillner, C. Staedler, V. Schoder, J. Welger, E. Bücheler and G. Adam, "Stellenwert der Dreiphasischen Spiral-CT in der Detektion des Hepatozellulären Karzinoms bei Leberzirrhose,” Röfo, Vol. 174, No. 1, 2002, pp. 96-100.

[16] T. Denecke, C. Grieser, V. Fröling, I. G. Steffen, B. Rudolph, L. Stelter, L. Lehmkuhl, F. Streitparth, J. Langrehr, P. Neuhaus and H. E. Lopez, "Multislice Computed Tomography Using a Triple-Phase Contrast Protocol for Preoperative Assessment of Hepatic Tumor Load in Patients with Hepatocellular Carcinoma before Liver Transplantation,” Transplant International, Vol. 22, No. 4, 2009, pp. 395-402. doi:10.1111/j.1432-2277.2008.00793.x

[17] M. Kanematsu, H. Hoshi, T. Yamada, T. Murakami, T. Kim, M. Kato, R. Yokoyama and H. Nakamura, "Small Hepatic Nodules in Cirrhosis: Ultrasonic, CT, and MR Imaging Findings,” Abdominal Imaging, Vol. 24, No. 1, 1999, pp. 47-55. doi:10.1007/s002619900439

[18] C. Bartolozzi, R. Lencioni, D. Caramella, A. Palla, A. M. Bassi and G. Di Candio, "Small Hepatocellular Carcinoma: Detection with US, CT, MR Imaging, DSA and Lipiodol-CT,” Acta Radiologica, Vol. 37, No. 1, 1996, pp. 69-74. doi:10.1080/02841859609174362

[19] T. Murakami, T. Kim and M. Takamura, "Hypervascular Hepatocellular Carcinoma: Detection with Double Arterial Phase Multi-Detector Row Helial CT,” Radiology, Vol. 218, No. 3, 2001, pp. 763-767.

[20] H. Oi, T. Murakami, T. Kim, M. Matsushita, H. Kishimoto and H. Nakamura, "Dynamic MR Imaging and Early-Phase Heliacal CT for Detection Small Intrahepatic Metastases of Hepatocellular Carcinoma,” American Journal of Roentgenology, Vol. 166, No. 2, 1996, pp. 369374. 
[21] H. R. Hentrich, L. Marti-Bonmati, A. Vanzulli, M. A. Kirchin, G. Piorovano, A. Spinazzi and K. P. Lodemann, "Charakterisierung Fokaler Leberläsionen: Wertigkeit von Multi-Hance (Gd-BOPTA) in der Kontrastverstärkten MRBildgebung,” Vortrag auf dem Deutschen Röntgenkongress, Wiesbaden, 2000.

[22] H. Horigome, T. Nomura, K. Saso, M. Itoh, T. Joh and H. Ohara, "Limitation of Imaging Diagnosis for Small Hepatocellular Carcinoma: Comparison with Histological Findings," Journal of Gastroenterology and Hepatology, Vol. 14, No. 6, 1999, pp. 559-565. doi:10.1046/j.1440-1746.1999.01915.x

[23] S. Jonas, T. Steinmüller, U. Settmacher, J. Langrehr, A. Müller and P. Neuhaus, "Liver Transplantation for Re- current Hepatocellular Carcinoma in Europe,” Journal of Hepato-Biliary-Pancreatic Surgery, Vol. 8, No. 5, 2001, pp. 422-426. doi:10.1007/s005340100004

[24] V. Mazzaferro, E. Regalia and R. Doci, "Liver Transplantation for the Treatment of Small Hepatocellular Carcinomas in Patients with Cirrhosis," The New England Journal of Medicine, Vol. 334, No. 11, 1996, pp. 693-699. doi:10.1056/NEJM199603143341104

[25] C. Benckert, S. Jonas, A. Thelen, A. Spinelli, G. Schumacher, M. Heise, J. Langrehr and P. Neuhaus, "Liver Transplantation for Hepatocellular Carcinoma in Cirrhosis: Prognostic Parameters,” Transplantation Proceedings, Vol. 37, No. 4, 2005, pp. 1693-1694. doi:10.1016/j.transproceed.2005.03.143 